\title{
Interacción genotipo-manejo de la alimentación en la producción de pollos camperos
}

\author{
Dottavio, A.M. ${ }^{1,3}$; Romera, B.M. ${ }^{1}$; Canet Z.E. ${ }^{1,2}$; Di Masso R.J.1,3 \\ ${ }^{1}$ Cátedra Genética, Fac.Cs.Vet.Univ.Nac.de Rosario (Casilda, Argentina). \\ ${ }^{2}$ INTA Pergamino. ${ }^{3} \mathrm{CIC}$ (Univ. Nac. Rosario-UNR). \\ E-mail: anadottavio@hotmail.com
}

\begin{abstract}
Resumen
Dottavio, A.M.; Romera, B.M.; Canet, Z.E.; Di Masso, R.J.: Interacción genotipo-manejo de la alimentación en la producción de pollos camperos. Rev. Vet. 30: 1, 43-47, 2019. La producción de carne aviar en sistemas de manejo semi-intensivo es una modalidad ecológica que contempla aspectos vinculados con el bienestar animal. El pollo Campero es un ave con menor velocidad de crecimiento que los parrilleros comerciales, destinado a la producción de carne. El esquema tradicional de alimentación incluye la utilización de tres tipos de alimentos especialmente formulados a tal fin (iniciador, crecimiento y terminador), lo cual introduce complicaciones en el manejo, particularmente en lo referido a la disponibilidad del alimento "crecimiento" para los pequeños productores. Una alternativa que facilitaría el manejo de estas aves es un esquema basado en sólo dos tipos de alimentos: iniciador y terminador. El objetivo de este trabajo fue evaluar el efecto del cambio del manejo tradicional de la alimentación por uno alternativo reemplazando el alimento "crecimiento" por una mezcla de iniciador y terminador, en dos genotipos de pollo Campero con diferente velocidad de crecimiento: el híbrido experimental de tres vías Campero Casilda y el híbrido simple Campero INTA. La evidencia indicó que el cambio en el manejo de la alimentación no afectó el crecimiento, la relación de conversión ni los caracteres a la faena. Ambos grupos genéticos se comportaron de manera equivalente, pudiendo obviarse la inclusión de la categoría "crecimiento" si se la reemplaza por una mezcla de iniciador y terminador.
\end{abstract}

Palabras clave: pollos, peso corporal, eficiencia alimenticia, caracteres a la faena, cruzamiento de tres vías.

\begin{abstract}
Dottavio, A.M.; Romera, B.M.; Canet, Z.E.; Di Masso, R.J.: Genotype x feeding regimen interactions in free-range chicken production. Rev. Vet. 30:1, 43-47, 2019. Poultry meat production in semi-intensive management systems is an ecological modality that takes into account aspects related to animal welfare. Campero chicken is a bird with a slower growth rate than commercial broilers destined to the production of meat in this type of systems. The traditional feeding scheme includes the use of three types of food specially formulated for this purpose (starter, grower and finisher) which introduces management complications, particularly in relation to the availability of grower food for small producers. An alternative that would facilitate feeding management is a scheme based on only two types of food: starter and finisher. The aim of this work was to evaluate the effect of changing the traditional feeding scheme by an alternative one, replacing grower food with a mix of starter and finisher, in two genotypes of chicken with a different growth rate: the experimental three-way cross Campero Casilda and the two-way cross Campero INTA. The evidence indicates that the proposed change in the traditional feeding management does not affect body growth, feed conversion ratio and productive traits at slaughter, and that both genetic groups behave similarly, when grower diet is replaced by a mix of starter and finisher.
\end{abstract}

Key words: chicken, body weight, feed efficiency, slaughter traits, three-way cross. 


\section{INTRODUCCIÓN}

La denominación avicultura alternativa hace referencia a cualquier modalidad de producción avícola que difiera de la convencional, e involucra sistemas menos intensivos con miras a la obtención de productos diferenciados de los tradicionales, por su calidad y/o por el modo de crianza de las aves, contemplando además aspectos vinculados con el bienestar animal ${ }^{3}$.

El pollo Campero INTA $^{1}$ es un tipo de ave destinada a la producción de carne en este tipo de sistemas, criado en base a un protocolo, en semi-cautiverio y con un esquema de alimentación que incluye la utilización de tres tipos de raciones: iniciador, crecimiento y terminador. Este pollo ha sido desarrollado como una alternativa para pequeños productores, donde las opciones genéticas comerciales muestran desajustes ante un ambiente no totalmente acorde a sus exigencias, permitiendo satisfacer una demanda creciente por productos naturales con un valor agregado diferencial .

Por intermedio del Programa Prohuerta este pollo es distribuido por el INTA Pergamino y una red de multiplicadores, para el autoconsumo de familias con necesidades alimentarias insatisfechas y venta de excedentes en ferias locales. El alimento crecimiento presenta problemas de disponibilidad en las diferentes zonas donde se distribuye -marginales para la avicultura industrial- por lo que su reemplazo implementando un manejo con sólo dos tipos de alimento, podría simplificar la cría de estas aves.

Dado que el alimento representa el principal componente del costo de producción y, en el caso del pollo Campero, la menor tasa de crecimiento de las aves conlleva a un deterioro de la relación de conversión alimenticia, las interacciones genotipo x manejo de alimentación adquieren relevancia por su impacto en la rentabilidad de las explotaciones dedicadas a producirlo.

El objetivo de este trabajo fue evaluar el efecto del cambio de manejo de la alimentación sobre las variables productivas habitualmente analizadas como salidas en estos sistemas de producción, en machos de pollo Campero derivados de dos modelos genéticos de cruzamientos terminales.

\section{MATERIAL Y MÉTODOS}

Se utilizaron pollos machos de los grupos genéticos: Campero Casilda y Campero INTA. Campero Casilda es un cruzamiento experimental de tres vías entre gallos de la sintética paterna AH' y gallinas derivadas del cruzamiento simple entre gallos y gallinas de las sintéticas maternas A y ES, respectivamente.

Campero INTA es un cruzamiento simple entre machos de la sintética paterna AS y hembras de la sintética materna E. Las poblaciones sintéticas intervinientes se generaron y mantienen en el Núcleo genético de la Sección Avicultura del INTA "Ing. Agr. Walter Kugler", en Pergamino (Argentina). La constitución genética teórica de las mismas (Bonino, comunicación personal) es:
Sintética materna A - 75\% Cornish Red $+25 \%$ Rhode Island Red; Sintética materna ES - 87,5\% Cornish Red $+12,5 \%$ Rhode Island Red, Sintética materna E - 50\% Cornish Red $+50 \%$ Rhode Island Red; Sintética paterna AH' - población mejorada por velocidad de crecimiento y eficiencia de conversión de alimento a partir de la sintética AH $[50 \%$ Hubbard $+50 \%$ estirpe Anak (grises)] y Sintética paterna AS - 50\% Cornish Red + 50\% Cornish Blanco.

El día del nacimiento, las aves se sexaron por inspección de la cloaca, se vacunaron contra la enfermedad de Marek, se identificaron con una banda alar numerada y se trasladaron a la Facultad de Ciencias Veterinarias de la Universidad Nacional de Rosario (Argentina) donde fueron sometidas al manejo y al plan sanitario establecido en el protocolo de certificación del pollo Campero INTA ${ }^{1}$.

Hasta los 35 días de edad se criaron a galpón como un grupo único con una densidad promedio de 15 aves/ $\mathrm{m}^{2} \mathrm{y}$ a partir del día 36 y hasta la faena a los 84 días de edad, permanecieron en galpones con acceso a parque con una densidad promedio de 8 aves $/ \mathrm{m}^{2}$ y 2 aves $/ \mathrm{m}^{2}$, respectivamente.

Se evaluaron dos manejos de alimentación: tradicional (MT- 40 aves de cada genotipo) basado en la utilización de tres tipos de alimento (Iniciador: 0 a 35 días de edad, Crecimiento: 36 a 56 días de edad y Terminador: 57 a 83 días de edad) y alternativo (MA- 40 aves de cada genotipo) basado en sólo dos tipos de alimento (Iniciador y Terminador, según el siguiente detalle: Iniciador (100\%) 0-28 días de edad; Iniciador $(75 \%)+$ Terminador (25\%) 29-42 días de edad; Iniciador (50\%) + Terminador (50\%) 43-56 días de edad; Iniciador (25\%) + Terminador (75\%) 57-70 días de edad y, por último, Terminador (100\%) 71-83 días de edad.

La formulación de las raciones se llevó a cabo según exigencias del protocolo mencionado, con la siguiente composición: Iniciador - proteína 18,5\%, calcio $0,9 \%$, fósforo total $0,75 \%$, fósforo disponible $0,47 \%$, energía metabolizable aves 3150 kcal., metionina + cistina $0,72 \%$ y lisina $0,94 \%$ y Terminador - proteína $15,09 \%$, calcio $0,85 \%$, fósforo total $0,6 \%$, fósforo disponible $0,38 \%$, energía metabolizable aves $3350 \mathrm{kcal}$, metionina + cistina $0,60 \%$ y lisina $0,75 \%$.

El peso de cada ave se registró a intervalos semanales entre el nacimiento y la faena. Los datos longitudinales peso corporal-edad se ajustaron por regresión no lineal con el modelo sigmoideo de Gompertz ${ }^{5}: \mathrm{W}(\mathrm{t})=$ $A \exp (-b \exp (-k t))$, donde: $W(t)=$ peso corporal $(g)$ en el tiempo $t, A=$ peso corporal asintótico (peso corporal promedio cuando $t$ tiende $a$ infinito), $b=$ parámetro de posición, constante de integración sin significado biológico, que ajusta para aquellos casos en que t es distinto de cero, $\mathrm{k}=$ tasa de maduración (velocidad de aproximación al valor A) y $\mathrm{t}=$ tiempo en semanas. La bondad del ajuste se evaluó a partir de la convergencia en una solución, el valor del coeficiente de determinación no lineal $\left(\mathrm{R}^{2}\right)$ y el comportamiento aleatorio de los residuales (test de rachas). 
A los efectos del tratamiento estadístico de los datos, los estimadores de los parámetros A y k se consideraron como nuevas variables aleatorias. A partir de los 36 días de vida, un grupo representativo de 40 aves de cada grupo genético (20 por cada manejo de la alimentación) se alojó en jaulas individuales. Luego de un período de siete días de acostumbramiento, cada ave fue pesada semanalmente, se calculó su aumento medio diario de peso (AMD) y se determinó su consumo medio diario individual de alimento (CMD) a partir del registro de la diferencia entre una cantidad fija ofrecida y la cantidad remanente en el comedero al día siguiente.

El comportamiento de la relación de conversión (RC:CMD/AMD) y las variables que la determinan se evaluó entre los 42 y 77 días de edad. Las aves se faenaron en la Sección Avicultura del INTA Pergamino (Argentina). En 30 aves de cada grupo genético y manejo de alimentación, se determinó el peso corporal pre-faena, el peso eviscerado, el peso de la pechuga con hueso y pata-muslo derecho y el peso del depósito graso abdominal como estimador del contenido de grasa corporal.

Los porcentajes de pechuga, pata-muslo y grasa abdominal se calcularon como proporción del peso corporal eviscerado. El rendimiento de la canal (\%) se calculó como [(peso corporal eviscerado / peso vivo pre-faena) x 100]. Los efectos del grupo genético, el manejo de la alimentación y la interacción simple entre ambos sobre las diferentes variables respuesta, se evaluaron con un análisis de la variancia correspondiente a un experimento factorial $2 \times 2$. Se consideraron significativos los efectos con probabilidades asociadas $\leq 0,05$.

\section{RESULTADOS}

La Tabla 1 presenta los valores de los estimadores de los parámetros de la función de Gompertz. Se observó convergencia en el ajuste, el coeficiente de de- terminación no lineal $\left(\mathrm{R}^{2}\right)$ superó el valor de 0,90 y los residuales mostraron una distribución aleatoria. No se observaron efectos significativos de la interacción grupo genético por manejo de la alimentación $(\mathrm{F}=0,34 ; \mathrm{P}=$ $0,558)$ sobre el valor promedio del estimador de peso corporal asintótico.

El grupo genético no representó una fuente significativa de variación con respecto a esta variable $(\mathrm{F}=$ 1,55; $\mathrm{P}=0,216)$ como así tampoco lo fue el manejo de la alimentación $(\mathrm{F}=0,85 ; \mathrm{P}=0,359)$. Con respecto a la tasa de maduración, la interacción tendió a ser significativa $(\mathrm{F}=2,93 ; \mathrm{P}=0,0898)$ debido a un mayor valor promedio del estimador en Campero Casilda con manejo tradicional y un menor valor del mismo en Campero INTA con manejo alternativo.

No se observó efecto significativo del manejo de la alimentación sobre la velocidad de aproximación al peso asintótico $(\mathrm{F}=0,11 ; \mathrm{P}=0,737)$ mientras que el grupo genético afectó significativamente a este carácter $(\mathrm{F}=27,9 ; \mathrm{P}<0,0001)$ correspondiendo valores mayores a las aves del cruzamiento experimental de tres vías.

La Tabla 2 presenta los valores de relación de conversión y variables asociadas que determinan su valor, en los dos genotipos, en cada manejo de alimentación.

No se observó efecto estadísticamente significativo de la interacción simple grupo genético $\mathrm{x}$ manejo de la alimentación sobre la $\mathrm{RC}(\mathrm{F}=0,10 ; \mathrm{P}=0,758)$, el $\operatorname{AMD}(\mathrm{F}=0,20 ; \mathrm{P}=0,656)$ y el $\mathrm{CMD}(\mathrm{F}=0,05 ; \mathrm{P}=0,817)$ lo que posibilitó la interpretación de los efectos directos de los factores principales. Tampoco se presentaron efectos estadísticamente significativos del grupo genético ( $\mathrm{RC}: \mathrm{F}=1,18 ; \mathrm{P}=0,282),(\mathrm{AMD}: \mathrm{F}=0,86 ; \mathrm{P}=0,357)$, (CMD: $\mathrm{F}=1,49 ; \mathrm{P}=0,226)$, ni del manejo de la alimentación ( $\mathrm{RC}: \mathrm{F}=0,00 ; \mathrm{P}=1,00),(\mathrm{AMD}: \mathrm{F}=0,00 ; \mathrm{P}=0,973)$, (CMD: $\mathrm{F}=0,04 ; \mathrm{P}=0,841$ ) sobre las tres variables analizadas.

Los valores de los caracteres estudiados a la faena se resumen en la Tabla 3.

Tabla 1. Estimadores de la función de Gompertz aplicada al ajuste de los datos peso corporal-edad cronológica en ambos genotipos.

\begin{tabular}{lcccc}
\hline & \multicolumn{2}{c}{ Campero Casilda } & \multicolumn{2}{c}{ Campero INTA } \\
& manejo tradicional & manejo alternativo & manejo tradicional & manejo alternativo \\
\hline peso asintótico A $(\mathrm{g})$ & $4777 \pm 92,5$ & $4917 \pm 88,1$ & $4947 \pm 94,0$ & $4978 \pm 96,6$ \\
tasa de maduración $\mathrm{k}\left(\mathrm{g}^{-1}\right)$ & $0,2227 \pm 0,00411$ & $0,2157 \pm 0,00349$ & $0,1988 \pm 0,00282$ & $0,2035 \pm 0,00312$ \\
\hline
\end{tabular}

Todos los valores corresponden a la media aritmética \pm error estándar. Tamaño muestral: $n=40$ aves por subgrupo genotipo-manejo de la alimentación.

Tabla 2. Relación de conversión y variables asociadas de dos genotipos de pollos Campero bajo dos manejos de la alimentación.

\begin{tabular}{lcccc}
\hline & \multicolumn{2}{c}{ Campero Casilda } & \multicolumn{2}{c}{ Campero INTA } \\
& manejo tradicional & manejo alternativo & manejo tradicional & manejo alternativo \\
\hline CMD (g/día) & $162,7 \pm 2,44$ & $164,1 \pm 4,15$ & $159,5 \pm 3,04$ & $159,4 \pm 3,06$ \\
AMD (g/día) & $49,9 \pm 1,28$ & $49,2 \pm 1,88$ & $47,9 \pm 1,25$ & $48,5 \pm 1,31$ \\
relac. conv. & $3,28 \pm 0,061$ & $3,37 \pm 0,086$ & $3,35 \pm 0,052$ & $3,30 \pm 0,053$ \\
\hline
\end{tabular}

relac.conv.: relación de conversión. Todos los valores corresponden a la media aritmética \pm error estándar. Tamaño muestral: $\mathrm{n}=20$ aves por subgrupo genotipo - manejo de la alimentación. 
Tabla 3. Caracteres a la faena en dos genotipos de pollos Campero bajo dos manejos de la alimentación.

\begin{tabular}{lcccc}
\hline & \multicolumn{2}{c}{ Campero Casilda } & \multicolumn{2}{c}{ Campero INTA } \\
& manejo tradicional & manejo alternativo & manejo tradicional & manejo alternativo \\
\hline peso prefaena $(\mathrm{g})$ & $3274 \pm 33,2$ & $3213 \pm 44,6$ & $3137 \pm 44,2$ & $3126 \pm 38,4$ \\
peso eviscerado $(\mathrm{g})$ & $2367 \pm 30,70$ & $2313 \pm 34,15$ & $2258 \pm 33,2$ & $2227 \pm 36,4$ \\
prop.de pechuga (\%) & $26,2 \pm 0,26$ & $26,0 \pm 0,41$ & $26,0 \pm 0,33$ & $25,6 \pm 0,21$ \\
prop. patamuslo (\%) & $15,9 \pm 0,39$ & $16,0 \pm 0,16$ & $15,3 \pm 0,16$ & $15,6 \pm 0,14$ \\
prop.grasa abdom.(\%) & $2,17 \pm 0,198$ & $2,08 \pm 0,146$ & $2,56 \pm 0,116$ & $2,28 \pm 0,309$ \\
rendimiento (\%) & $72,3 \pm 0,30$ & $72,0 \pm 0,24$ & $72,0 \pm 0,24$ & $71,2 \pm 0,31$ \\
\hline
\end{tabular}

Todos los valores corresponden a la media aritmética \pm error estándar. Tamaño muestral: $\mathrm{n}=30$ aves por subgrupo genotipo - manejo de la alimentación.

No se observaron efectos estadísticamente significativos de la interacción grupo genético $\mathrm{x}$ manejo de la alimentación sobre ninguno de los caracteres evaluados. El efecto del grupo genético fue estadísticamente significativo para el peso corporal pre-faena $(\mathrm{F}=7,68$; $\mathrm{P}=0,007)$, el peso eviscerado $(\mathrm{F}=11,6 ; \mathrm{P}=0,001)$ y el rendimiento a la faena $(\mathrm{F}=3,56 ; \mathrm{P}=0,063)$.

Si bien las aves Campero Casilda tendieron a mostrar mayor proporción de pechuga (Campero Casilda: $26,1 \%$ vs Campero INTA: $25,8 \%$ ) y menor proporción de grasa abdominal (Campero Casilda: $2,13 \%$ vs Campero INTA: $2,42 \%$ ) dichas diferencias no alcanzaron significado estadístico. Sólo se observó efecto estadísticamente significativo del manejo de la alimentación en el caso del rendimiento a la faena $(\mathrm{F}=3,81 ; \mathrm{P}=0,055)$ que resultó mayor bajo manejo tradicional.

\section{DISCUSIÓN}

De acuerdo a especialistas en el tema ${ }^{10}$, la expresión interacción genotipo $\mathrm{x}$ ambiente se utiliza para describir aquellas situaciones en las cuales diferentes genotipos responden de manera diferencial ante un cambio de ambiente, lo que podría atribuirse a variaciones relativas en las contribuciones individuales $o$ niveles de expresión de los genes involucrados en la determinación de un carácter.

La producción de carne de cualquier especie de interés económico se basa en el crecimiento de los individuos utilizados, proceso afectado por el ambiente nutricional y factores hormonales con funciones regulatorias que intervienen en la conversión de los nutrientes y en sus modalidades de almacenamiento ${ }^{4}$.

Dado que el balance hormonal depende de factores genéticos y también de la dieta de las aves, la combinación de ambos puede dar lugar a interacciones significativas. Diferentes autores han investigado la presencia de interacciones genotipo $\mathrm{x}$ dieta y sus efectos sobre algunos caracteres productivos en parrilleros comerciales ${ }^{7,12}$.

Los genotipos evaluados en este trabajo difieren en relación a la estrategia genética utilizada para generarlos y en su velocidad de crecimiento, lo cual dado el esquema simplificado de alimentación propuesto, podría derivar en interacciones genotipo $\mathrm{x}$ manejo de la alimentación. La caracterización del patrón de creci- miento de estos genotipos cobra importancia dada su condición de aves destinadas a la producción de carne y su dinámica resulta de la combinación entre el peso asintótico y la tasa de aproximación al mismo.

Pese a crecer hacia pesos asintóticos similares, la mayor tasa de maduración del cruzamiento experimental de tres vías determina que, independientemente del manejo de la alimentación, las aves de este grupo genético sean ligeramente más pesadas que las del genotipo de referencia a la edad de faena. En relación a la conversión de alimento en biomasa, su caracterización adquiere relevancia debido a particularidades que diferencian a las poblaciones de crecimiento lento de aquellas empleadas en la avicultura industrial.

La menor tasa de aumento de peso diario observada respecto de las líneas comerciales y una edad de faena que prácticamente duplica a la del broiler industrial, contribuyen a un deterioro de la conversión de alimento en carne.

De todos modos, en estos sistemas productivos la eficiencia no siempre es evaluada en términos de máxima ganancia de peso y conversión ${ }^{6}$ debiendo considerarse otras variables tales como la modalidad de crianza en relación a demandas particulares del mercado consumidor y la calidad del producto ofertado ${ }^{11}$ que en algunos casos justifican llegar con mayores pesos de faena ${ }^{9} \mathrm{e}$ incluso no estar sujetos a lograr un objetivo de conversión sino a considerar en el análisis todas las variables que determinan la mejor relación costo/ beneficio ${ }^{2}$.

Los valores de relación de conversión observados en este estudio, oscilaron entre un máximo de 3,35 para Campero INTA y un mínimo de 3,28 en Campero Casilda, ambos criados con manejo tradicional. Si bien los mismos no difieren de los informados en estudios previos llevados a cabo en cruzamientos experimentales de crecimiento más lento y se encuentran dentro del rango comunicado para pollos Label Rouge por otros autores ${ }^{8}$, superan a los habituales en el modelo productivo intensivo, lo que implica mayores costos de alimentación y, en consecuencia, un mayor precio de venta del producto final.

Por último, y en relación a la proporción de cortes valiosos y de rendimiento a la faena, los valores observados son los esperados en este tipo de aves de crecimiento más lento. Considerando la propuesta de 
simplificación del esquema de alimentación establecido en el protocolo, tanto en Campero Casilda como en Campero INTA, el alimento crecimiento utilizado en el manejo tradicional puede ser reemplazado por una combinación de alimentos iniciador y terminador sin afectar la proporción de cortes valiosos ni el contenido de grasa corporal, si bien con un leve efecto detrimental sobre el rendimiento a la faena.

La evidencia indica que los dos grupos genéticos se comportan de manera equivalente en relación a las variables analizadas y que el cambio propuesto en el manejo de la alimentación, en términos generales, no afecta a los caracteres productivos evaluados pudiendo obviarse la inclusión de la categoría crecimiento en el esquema, simplificando así su producción dado que estas aves reciben alimento balanceado especialmente formulado para pollos Campero.

Agradecimientos. A los estudiantes de Veterinaria de la UNR por colaborar responsablemente en el cuidado de los animales y en el registro de los datos, en particular a Sabina Advínculo, Araceli Martines y José Librera.

\section{REFERENCIAS}

1. Bonino MF, Canet ZE. 1999. Producción de pollos y huevos camperos. Boletín Técnico editado por la Dirección de Comunicaciones INTA Pergamino, $39 \mathrm{p}$.

2. Buxade CC. 1988. El pollo de carne, 2da. ed., Mundi Prensa, Madrid, $365 \mathrm{p}$

3. Castelló JA. 2002. La industria del pollo para carne. En: Producción de carne de pollo (Castelló JA, Cedó R, Cepero R, et al), Ed.Amazon), México, 425 p.
4. Decuypere E. 2007. The endocrine and metabolic interface of genotype-nutrition interaction in broilers and broiler breeders. World's Poult Sci J 63: 115-128.

5. Fitzhugh H. 1976. Analysis of growth curves and strategies for altering their shape. J Anim Sci 42: 1036-1051.

6. Fumero JE, Godínez O, Arias R. 2010. Prueba comparativa de pollos híbridos camperos y comerciales en crianza intensiva. Rev Cubana Ciencia Avíc 34: 29-36.

7. Havenstein GB, Ferket PR, Qureshi MA. 2003. Growth, livability and feed conversion of 1957 versus 2001 broilers when fed representative 1957 and 2001 broiler diets. Poult Sci 82: 1509-1518.

8. N'Dri A, Mignon GS, Sellier N, Tixier BM, Beaumont C. 2006. Genetic relationships between feed conversion ratio, growth curve and body composition in slow-growing chickens. Br Poult Sci 47: 273-280.

9. North MO. 1993. Manual de Producción avícola, Ed. Manual Moderno, 3ra. ed., México, 829 p.

10. Sheridan A. 1990. Genotype x environment interactions. In: Poultry breeding and genetics (Crawford RD Ed), Elsevier, New York, p. 897-912.

11. Terraes JC et al. 2007. Efectos de dos dietas de diferente composición sobre el porcentaje de uniformidad de pollos parrilleros hembras. Anales XXVIII Sesión Comunic. Científ., Fac. Cs. Veterinarias (Corrientes, Argentina), p. 47.

12. Whitehead C, Griffin H. 1984. Development of divergent lines of lean and fat broilers using plasma very low density lipoprotein concentrations as selection criterion: the first three generations. Br Poult Sci 25: 573-582. 